\title{
Total positivity for cominuscule Grassmannians
}

\author{
Thomas $\operatorname{Lam}^{1 \dagger}$ and Lauren Williams ${ }^{1}$ \\ ${ }^{1}$ Department of Mathematics, Harvard University, Cambridge MA 02138 USA
}

\begin{abstract}
In this paper we explore the combinatorics of the non-negative part $(G / P)_{\geq 0}$ of a cominuscule Grassmannian. For each such Grassmannian we define $\amalg$-diagrams - certain fillings of generalized Young diagrams which are in bijection with the cells of $(G / P)_{\geq 0}$. In the classical cases, we describe I-diagrams explicitly in terms of pattern avoidance. We also define a game on diagrams, by which one can reduce an arbitrary diagram to a J-diagram. We give enumerative results and relate our $\amalg$-diagrams to other combinatorial objects. Surprisingly, the totally nonnegative cells in the open Schubert cell of the odd and even orthogonal Grassmannians are (essentially) in bijection with preference functions and atomic preference functions respectively.
\end{abstract}

Résumé. Dans cet article nous schtroumpfons la combinatoire de la partie non-négative $(G / P)_{\geq 0}$ d'une Grassmannienne cominuscule. Pour chaque Grassmannienne de ce type nous définissons les I-diagrammes - certains remplissages de diagrammes de Young généralisés en bijection avec les cellules de $(G / P)_{\geq 0}$. Dans les cas classiques, nous décrivons les I-diagrammes explicitement en termes d'évitement de certains motifs. Aussi nous définissons un jeu sur les diagrammes, avec lequel on peut réduire un diagramme arbitraire à un J-diagramme. Nous donnons les résultats énumératifs et nous relions nos J-diagrammes à d'autres objets combinatoires. Etonnamment, les cellules non-négatives dans la cellule de Schubert ouverte des Grassmanniennes orthogonales impaires et paires sont essentiellement en bijection avec les fonctions de préférence et les fonctions de préférence atomiques.

Keywords: Total positivity, Grassmannian, tableaux, preference functions

\section{Introduction}

The classical theory of total positivity concerns matrices in which all minors are non-negative. While this theory was pioneered in the 1930's, interest in this subject has been renewed on account of the work of Lusztig (6). Motivated by surprising connections he discovered between his theory of canonical bases for quantum groups and the theory of total positivity, Lusztig extended this subject by introducing the totally non-negative points $G_{\geq 0}$ in an arbitrary reductive group $G$ and the totally non-negative part $(G / P)_{\geq 0}$ of

\footnotetext{
${ }^{\dagger}$ NSF DMS-0600677

1365-8050 (C) 2008 Discrete Mathematics and Theoretical Computer Science (DMTCS), Nancy, France
} 
a real flag variety $G / P$. Lusztig conjectured a cell decomposition for $(G / P)_{\geq 0}$, which was proved by Rietsch (11). Cells of $(G / P)_{\geq 0}$ correspond to pairs $(x, w)$ where $x, w \in W, x \leq w$ in Bruhat order, and $w$ is a minimal-length coset representative of $W^{J}=W / W_{J}$. Here $W_{J} \subset W$ is the parabolic subgroup corresponding to $P$.

With a more combinatorial perspective, Postnikov (8) explored the combinatorics of the totally nonnegative part of the type $A$ Grassmannian. He described and parameterized cells using certain fillings of Young diagrams by 0's and +'s which he called I-diagrams, and which are defined using the avoidance of the J-pattern. The I-diagrams seem to have a great deal of intrinsic interest: they were independently discovered by Cauchon (2) in the context of primes in quantum algebras; they are in bijection with other combinatorial objects (8); and they are linked to the asymmetric exclusion process (3).

In this paper we use work of Stembridge (14) and Proctor (10) to generalize J-diagrams to cominuscule Grassmannians. In this case the poset $W^{J}$ can be identified with the lattice of order ideals of another poset $Q^{J}$; moreover, $Q^{J}$ can always be embedded into a two-dimensional square lattice. Each $w \in W^{J}$ corresponds to an order ideal $O_{w} \subset Q^{J}$ which can be represented by a generalized Young diagram. We identify cells of the non-negative part of a cominuscule Grassmannian with certain fillings, called I-diagrams, of $O_{w}$ by 0's and +'s. Arbitrary fillings of $O_{w}$ by 0 's and +'s correspond to subexpressions of a reduced expression for $w$; the I-diagrams correspond to positive distinguished subexpressions (7).

We give concise descriptions of $J$-diagrams for type $B$ and $D$ cominuscule Grassmannians in terms of pattern avoidance. Unfortunately there does not seem to exist a concise description for the remaining $E_{6}$ and $E_{7}$ cominuscule Grassmannians. We also define a game (the I-game) that one can play on diagrams filled with 0's and +'s, by which one can go from any such diagram to a J-diagram.

We then give some formulas and recurrences for the numbers of I-diagrams. We also show that there are twice as many type $\left(B_{n}, n\right) \mathrm{J}$-diagrams in the open Schubert cell as preference functions of length $n$, while type $\left(D_{n}, n\right)$ I-diagrams in the open Schubert cell are in bijection with atomic preference functions of length $n$. We can also define other related combinatorial objects, such as decorated permutations and permutation tableaux, but for lack of space these are not treated in this abstract.

\section{Total positivity for flag varieties}

Let $G$ be a semisimple linear algebraic group over $\mathbb{C}$ split over $\mathbb{R}$, with split torus $T$. Identify $G$ (and related spaces) with their real points and consider them with their real topology. Let $\Phi \subset \operatorname{Hom}\left(T, \mathbb{R}^{*}\right)$ the set of roots and choose a system of positive roots $\Phi^{+}$. Denote by $B^{+}$the Borel subgroup corresponding to $\Phi^{+}$. Let $B^{-}$be the opposite Borel subgroup $B^{-}$such that $B^{+} \cap B^{-}=T$. Let $U^{+}$and $U^{-}$be the unipotent radicals of $B^{+}$and $B^{-}$.

Denote the set of simple roots by $\Pi=\left\{\alpha_{i} \mid i \in I\right\} \subset \Phi^{+}$. For each $\alpha_{i} \in \Pi$ there is an associated homomorphism $\phi_{i}: \mathrm{SL}_{2} \rightarrow G$, generated by 1-parameter subgroups $x_{i}(t) \in U^{+}, y_{i}(t) \in U^{-}$, and $\alpha_{i}^{\vee}(t) \in T$. The datum $\left(T, B^{+}, B^{-}, x_{i}, y_{i} ; i \in I\right)$ for $G$ is called a pinning. Let $W=N_{G}(T) / T$ be the Weyl group and for $w \in W$ let $\dot{w} \in N_{G}(T)$ denote a representative for $w$.

Let $J \subset I$. The parabolic subgroup $W_{J} \subset W$ corresponds to a parabolic subgroup $P_{J}$ in $G$ containing $B^{+}$. Namely, $P_{J}=\sqcup_{w \in W_{J}} B^{+} \dot{w} B^{+}$. Let $\pi^{J}: G / B^{+} \rightarrow G / P_{J}$ be the natural projection. 
The totally non-negative part $U_{>0}^{-}$of $U^{-}$is defined to be the semigroup in $U^{-}$generated by the $y_{i}(t)$ for $t \in \mathbb{R}_{\geq 0}$. The totally non-negative part $\left(G / P_{J}\right)_{\geq 0}$ of the partial flag variety $G / P_{J}$ is the closure of the image of $U_{>0}^{-}$in $G / P_{J}$.

We have the Bruhat decompositions

$$
G / B^{+}=\sqcup_{w \in W} B^{+} \dot{w} B^{+} / B^{+}=\sqcup_{w \in W} B^{-} \dot{w} B^{+} / B^{+}
$$

of $G / B^{+}$into $B^{+}$-orbits called Bruhat cells, and $B^{-}$-orbits called opposite Bruhat cells. For $v, w \in W$ define

$$
R_{v, w}:=B^{+} \dot{w} B^{+} / B^{+} \cap B^{-} \dot{v} B^{+} / B^{+} .
$$

The intersection $R_{v, w}$ is non-empty precisely if $v \leq w$, and in that case is irreducible of dimension $\ell(w)-\ell(v)$. Here $\leq$ denotes the Bruhat order (or strong order) of $W(1)$. For $v, w \in W$ with $v \leq w$, let

$$
R_{v, w ;>0}:=R_{v, w} \cap\left(G / B^{+}\right) \geq 0 .
$$

We write $W^{J}$ for the set of minimal length coset representatives of $W / W_{J}$. The Bruhat order of $W^{J}$ is the order inherited by restriction from $W$. Let $\mathcal{I}^{J} \subset W \times W^{J}$ be the set of pairs $(x, w)$ with the property that $x \leq w$. Given $(x, w) \in \mathcal{I}^{J}$, we define $P_{x, w ;>0}^{J}:=\pi^{J}\left(R_{x, w ;>0}\right)$. This decomposition of $\left(G / P_{J}\right)_{\geq 0}$ was introduced by Lusztig (6). Rietsch showed that the sets $P_{x, w ;>0}^{J}$ are semi-algebraic cells of dimension $\ell(w)-\ell(x)$, giving a cell decomposition of $\left(G / P_{J}\right)_{\geq 0}(11)$. In fact the cell decomposition is a CW complex $(9 ; 12)$.

\section{3 (Co)minuscule Grassmannians}

We keep the notation of Section 2. We say the parabolic $P_{J}$ is maximal if $J=I \backslash\{j\}$ for some $j \in I$. We may then denote the parabolic by $P_{j}:=P_{J}$ and the partial flag variety by $G / P_{j}:=G / P_{J}$, which we loosely call a Grassmannian. Similarly, we use the notation $\mathcal{I}^{j}, W^{j}, W_{j}$ and $W_{\max }^{j}$.

For a maximal parabolic subgroup $P_{j}$ we will call $P_{j}$, the flag variety $G / P_{j}$, and the simple root $\alpha_{j}$ cominuscule if whenever $\alpha_{j}$ occurs in the simple root expansion of a positive root $\gamma$ it does so with coefficient one. Similarly, one obtains the definition of minuscule by replacing roots with coroots. The (co)minuscule Grassmannian's have been classified and are listed below, with the corresponding Dynkin diagrams (plus choice of simple root) shown in Figure 1.

Proposition 3.1 The maximal parabolic $P_{j}$, the flag variety $G / P_{j}$, and the simple root $\alpha_{j}$ are (co)minuscule if we are in one of the following situations:

1. $W=A_{n}$ and $j \in[1, n]$ is arbitrary

2. $W=B_{n}\left(\right.$ or $\left.C_{n}\right)$ and $j=1$ or $n$

3. $W=D_{n}$ (with $n \geq 4$ ) and $j=1, n-1$ or $n$

4. $W=E_{6}$ and $j=1$ or 6 


\begin{tabular}{|c|c|c|}
\hline Root system & Dynkin Diagram & Grassmannian \\
\hline$A_{n}$ & 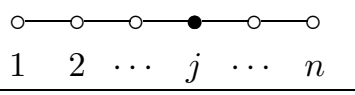 & the usual Grassmannian $\mathrm{Gr}_{j, n+1}$ \\
\hline$B_{n}, n \geq 2$ & $\begin{array}{lllll}\bullet & 0 & 0 & 0 & 0 \\
1 & 2 & \cdots & \cdots & n\end{array}$ & the odd dimensional quadric $\mathbb{Q}^{2 n-1}$ \\
\hline$B_{n}, n \geq 2$ & $\begin{array}{lllll}\circ & 0 & \cdots & \cdots & \cdots \\
1 & 2 & \cdots & \cdots & n\end{array}$ & odd orthogonal Grassmannian $\mathrm{OG}_{n, 2 n+1}$ \\
\hline$C_{n}, n \geq 2$ & $\begin{array}{lllll}1 & 2 & \cdots & \cdots & n \\
\end{array}$ & the projective space $\mathbb{P}^{2 n-1}$ \\
\hline$C_{n}, n \geq 2$ & 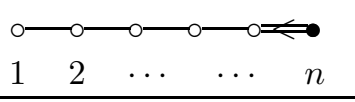 & the Lagrangian Grassmannian $\mathrm{LG}_{n, 2 n}$ \\
\hline$D_{n}, n \geq 4$ & $\begin{array}{llllll}1 & 2 & \cdots & \cdots & n-1\end{array}$ & the even dimensional quadric $\mathbb{Q}^{2 n-2}$ \\
\hline$D_{n}, n \geq 4$ & $\begin{array}{lllll}1 & 2 & \cdots & \cdots & n-1\end{array}$ & even orthogonal Grassmannian $\mathrm{OG}_{n+1,2 n+1}$ \\
\hline$E_{6}$ & $\begin{array}{llllll} & & & 9 & & \\
\bullet & & & & & \\
1 & 3 & 4 & 5 & 6\end{array}$ & the real points of the Cayley plane $\mathbb{O P}^{2}$ \\
\hline$E_{7}$ & 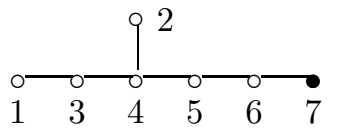 & the (real) Freudenthal variety Fr \\
\hline
\end{tabular}

Fig. 1: The (co)minuscule parabolic quotients

5. $W=E_{7}$ and $j=1$.

Besides the Bruhat (strong) order, we also have the weak order on a parabolic quotient (see (1) for details). An element $w \in W$ is fully commutative if every pair of reduced words for $w$ are related by a sequence of relations of the form $s_{i} s_{j}=s_{j} s_{i}$. The following result is due to Stembridge (14) (part of the statement is due to Proctor (10)).

Theorem 3.2 If $(W, j)$ is (co)minuscule then $W^{j}$ consists of fully commutative elements. Furthermore the weak order $\left(W^{j}, \prec\right)$ and strong order $\left(W^{j},<\right)$ of $W^{j}$ coincide, and this poset is a distributive lattice.

Since $\left(W^{j}, \prec\right)$ and $\left(W^{j},<\right)$ coincide, we will just refer to this partial order as $W^{j}$. We indicate in Figure 2 (mostly taken from (5)) the posets $Q^{j}$ such that $W^{j}=J\left(Q^{j}\right)$, where $J(P)$ denotes the distributive lattice of order ideals in $P$. Note that the posets are drawn in "French" notation so that minimal elements are at the bottom left. The diagrams should be interpreted as follows: each box represents an element of the poset $Q^{j}$, and if $b_{1}$ and $b_{2}$ are two adjacent boxes such that $b_{1}$ is immediately to the left or 
immediately below $b_{2}$, we have a cover relation $b_{1} \lessdot b_{2}$ in $Q^{j}$. The partial order on $Q^{j}$ is the transitive closure of $\lessdot$. (In particular the labeling of boxes shown in Figure 2 does not affect the poset structure.)

We now state some facts about $Q^{j}$ which can be found in (14). Let $w_{0}^{j} \in W^{j}$ denote the longest element in $W^{j}$. The simple generators $s_{i}$ used in a reduced expression for $w_{0}^{j}$ can be used to label $Q^{j}$ in a way which reflects the bijection between the minimal length coset representatives $w \in W^{j}$ and (lower) order ideals $O_{w} \subset Q^{j}$. Such a labeling is shown in Figure 2; the label $i$ stands for the simple reflection $s_{i}$. If $b \in O_{w}$ is a box labelled by $i$, we denote the simple generator labeling $b$ by $s_{b}:=s_{i}$; the corresponding index $i \in I$ is the simple label of $b$.

Given this labeling, if $O_{w}$ is an order ideal in $Q^{j}$, the set of linear extensions $\left\{e: O_{w} \rightarrow[1, \ell(w)]\right\}$ of $O_{w}$ are in bijection with the reduced words $R(w)$ of $w$ : the reduced word (written down from right to left) is obtained by reading the labels of $O_{w}$ in the order specified by $e$. We will call the linear extensions of $O_{w}$ reading orders. (Alternatively, one may think of a linear extension of $O_{w}$ as a standard tableau with shape $O_{w}$.)

\section{I-diagrams, I-moves and the J-game}

\subsection{Positive distinguished subexpressions}

Consider a reduced expression in $W$, say $s_{3} s_{2} s_{1} s_{3} s_{2} s_{3}$ in type $A_{3}$. We define a subexpression to be a word obtained from a reduced expression by replacing some of the factors with 1. For example, $s_{3} s_{2} 1 s_{3} s_{2} 1$ is a subexpression of $s_{3} s_{2} s_{1} s_{3} s_{2} s_{3}$. Given a reduced expression $\mathbf{w}:=s_{i_{1}} s_{i_{2}} \ldots s_{i_{n}}$ for $w$, we set $w_{(k)}:=$ $s_{i_{1}} s_{i_{2}} \ldots s_{i_{k}}$ if $k \geq 1$ and $w_{(0)}=1$. The following definition was given in (7) and was implicit in (4).

Definition 4.1 (Positive distinguished subexpressions) Let $\mathbf{w}:=s_{i_{1}}, \ldots, s_{i_{n}}$ be a reduced expression. We call a subexpression $\mathbf{v}$ of $\mathbf{w}$ positive distinguished if

$$
v_{(j-1)}<v_{(j-1)} s_{i_{j}}
$$

for all $j=1, \ldots, n$.

Note that (1) is equivalent to $v_{(j-1)} \leq v_{(j)} \leq v_{(j-1)} s_{i_{j}}$. We will refer to a positive distinguished subexpression as a PDS for short. The following lemma is from (7).

Lemma 4.2 Given $v \leq w$ in $W$ and a reduced expression $\mathbf{w}$ for $w$, there is a unique PDS $\mathbf{v}_{+}$for $v$ in $\mathbf{w}$.

\section{$4.2 \oplus$-diagrams and I-diagrams}

The goal of this section is to identify the PDS's with certain fillings of the boxes of order ideals of $Q^{j}$.

Let $O_{w}$ be an order ideal of $Q^{j}$, where $w \in W^{j}$.

Definition 4.3 An $\oplus$-diagram ("o-plus diagram") of shape $O_{w}$ is a filling of the boxes of $O_{w}$ with the symbols 0 and + . 
Clearly there are $2^{\ell(w)} \oplus$-diagrams of shape $O_{w}$. The value of an $\oplus$-diagram $D$ at a box $x$ is denoted $D(x)$. Let $e$ be a reading order for $O_{w}$; this gives rise to a reduced expression $\mathbf{w}=\mathbf{w}_{e}$ for $w$. The $\oplus$-diagrams $D$ of shape $O_{w}$ are in bijection with subexpressions $\mathbf{v}(D)$ of $\mathbf{w}$ : we will make the seemingly unnatural specification that if a box $b \in O_{w}$ is filled with a 0 then the corresponding simple generator $s_{b}$ is present in the subexpression, while if $b$ is filled with a + then we omit the corresponding simple generator. The subexpression $\mathbf{v}(D)$ in turn defines a Weyl group element $v:=v(D) \in W$.

Example 4.4 Consider the order ideal $O_{w}$ which is $Q^{j}$ itself for type $A_{4}$ with $j=2$. Then $Q^{j}$ is the following poset

$$
\begin{array}{|l|l|l|}
\hline 1 & 2 & 3 \\
\hline 2 & 3 & 4 \\
\hline
\end{array}
$$

Let us choose the reading order (linear extension) indicated by the labeling below:

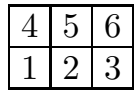

Then the $\oplus$-diagrams

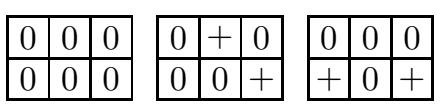

correspond to the expressions $s_{3} s_{2} s_{1} s_{4} s_{3} s_{2}, s_{3} 1 s_{1} 1 s_{3} s_{2}$ and $s_{3} s_{2} s_{1} 1 s_{3} 1$. The first and the last are PDS's while the second one is not, since it is not reduced.

Proposition 4.5 Let $D$ be an $\oplus$-diagram. Then

1. the element $v:=v(D)$ is independent of the choice of reading word $e$.

2. whether $\mathbf{v}(D)$ is a PDS depends only on $D$ (and not $e$ ).

Proposition 4.5 allows us to make the following definition.

Definition 4.6 $A$ I-diagram of shape $O_{w}$ is an $\oplus$-diagram $D$ of shape $O_{w}$ such that $\mathbf{v}(D)$ is a PDS.

Theorem 4.7 The cells of $\left(G / P_{j}\right)_{\geq 0}$ are in bijection with pairs $\left(D, O_{w}\right)$ where $O_{w}$ is an order ideal in $Q^{j}$ and $D$ is a $\mathrm{I}$-diagram of shape $O_{w}$. Furthermore, the cell labeled by $\left(D, O_{w}\right)$ is isomorphic to $\left(\mathbb{R}^{+}\right)^{s}$ where $s$ is the number of + 's in $D$.

Problem 4.8 Give a compact description of I-diagrams.

\subsection{The I-game}

Let $D$ be an $\oplus$-diagram of shape $O_{w}$ corresponding to an element $v(D) \in W$. By Lemma 4.2 and Proposition 4.5 there is a unique I-diagram $D_{+}$with $v\left(D_{+}\right)=v(D)$. We call $D_{+}$the $\mathrm{I}$-ification of $D$.

Problem 4.9 Describe how to produce $D_{+}$from $D$. 
Our solution to Problem 4.9 will be algorithmic, involving a series of game-like moves. Suppose $C \subset O_{w}$ is a convex subset: that is, if $x$ and $y$ are in $C$ then any $z$ such that $x<z<y$ must also be in $C$. We may extend the definition of $\oplus$-diagrams to $C$. In addition Proposition 4.5 still holds for $\oplus$-diagrams of shape $C$. If $D$ is an $\oplus$-diagram of shape $C$ we again denote by $v(D) \in W$ the corresponding Weyl group element. If $S: C \rightarrow\{0,+, ?\}$ is a filling of $C$ with the symbols $0,+$ and ?, we say that an $\oplus$-diagram $D$ is compatible with $S$ if for every $x \in C$

1. $D(x)=0 \Longrightarrow S(x) \in\{0, ?\}$, and

2. $D(x)=+\Longrightarrow S(x) \in\{+, ?\}$.

If $x, y \in O_{w}$ are two boxes we let $(x, y)=\left\{z \in O_{w} \mid x<z<y\right\}$ be the open interval between $x$ and $y$. Similarly, define the half open intervals $(x, y]$ and $[x, y)$.

Definition 4.10 $A$ I-move $M$ is a triple $(x, y, S)$ consisting of a pair $x<y \in O_{w}$ of comparable, distinct boxes together with a filling of the open interval $S:(x, y) \rightarrow\{0,+, ?\}$ such that

$$
v(D \cup x)=v(D \cup y)
$$

for every $\oplus$-diagram $D$ of shape $(x, y)$ compatible with $S$. Here $D \cup x(D \cup y)$ is the $\oplus$-diagram of shape $[x, y)((x, y])$ obtained from $D$ by placing $a 0$ in $x(y)$. We say that $(x, y, S)$ is a I-move from $y$ to $x$ via $S$.

Now if $D$ is an $\oplus$-diagram whose shape contains $[x, y]$, we say that a I-move $M=(x, y, S)$ can be performed on $D$ if $D(y)=0$ and $\left.D\right|_{(x, y)}$ is compatible with $S$. The result of $M$ on $D$ is then the $\oplus$ diagram $D^{\prime}$ obtained from $D$ by setting $D(y)=+$ and switching the entry of $D(x)$ (that is, $D^{\prime}(x)=0$ if $D(x)=+$ and $D^{\prime}(x)=+$ if $\left.D(x)=0\right)$.

For two $\{0,+, ?\}$-fillings $S, S^{\prime}$ of the same shape let us say that $S^{\prime}$ is a specialization of $S$ (and $S$ a generalization of $S^{\prime}$ ) if $S^{\prime}$ is obtained from $S$ by changing some ?'s to 0's or +'s. It is then clear from the definition that if $S^{\prime}$ is a specialization of $S$ and $(x, y, S)$ is a I-move then so is $\left(x, y, S^{\prime}\right)$.

Performing a $J$-move on an $\oplus$-diagram $D$ either reduces the number of 0 's or moves a 0 to a box which is smaller in the partial order (and the + to a bigger box). Thus any sequence of I-moves must eventually terminate.

Proposition 4.11 No J-moves can be performed on a I-diagram. Every $\oplus$-diagram $D$ can be $\mathrm{I}$-ified by a finite sequence of $\mathrm{I}$-moves.

We say a set $\mathbb{S}$ of I-moves is complete if every $\oplus$-diagram $D$ can be I-ified using I-moves in $\mathbb{S}$.

Problem 4.12 Describe a complete set of I-moves.

\section{Description of I-diagrams}

In this section we will give compact descriptions of I-diagrams in types $A, B$, and $D$. In each case we have complete sets of I-moves, but for lack of space we will only include them in type $A$. 
First let $(W, j)=\left(A_{n-1}, j\right)$ so that any $O_{w}$ can be identified with a Young diagram within a $j \times(n-j)$ rectangle.

The following theorem shows that our (more general) definition of I-diagram agrees with Postnikov's definition of I-diagram when one is working in type A.

Theorem 5.1 An $\oplus$-diagram of shape $O_{w}$ in type $A_{n-1}$ is a I-diagram if and only if there is no 0 which has $a+$ below it and $a+$ to its left.

In Theorem 5.1, "below" means below and in the same column, while "to its left" means to the left and in the same row. If an $\oplus$-diagram satisfies these condition, we say that it possesses the $\mathrm{J}$-condition.

Let $x<y$ be two distinct, comparable boxes in $O_{w}$. Then $[x, y]$ is a rectangle (or as a poset, a product of chains). Given $x<y$, let $S_{0}$ denote the following $\{0,+, ?\}$ filling of $(x, y)$ :

\begin{tabular}{|c|c|c|c|c|}
\hline+ & 0 & 0 & 0 & $y$ \\
\hline 0 & 0 & 0 & 0 & 0 \\
\hline 0 & 0 & 0 & 0 & 0 \\
\hline$x$ & 0 & 0 & 0 & + \\
\hline
\end{tabular}

That is, $S_{0}$ is filled with 0's except for the top left and bottom right corners, where it is filled with +'s.

Proposition 5.2 The triples $\left(x, y, S_{0}\right)$ defined above form a complete system of $\mathrm{I}$-moves.

In (8), Postnikov studied the totally non-negative part of the type A Grassmannian $\left(G r_{k, n}\right)_{\geq 0}$, and showed that it has a cell decomposition where cells are in bijection with certain combinatorial objects he called J-diagrams. Postnikov's I-diagrams are obtained from ours by reflecting in a horizontal axis. Since Postnikov was using the English convention for Young diagrams whereas we are using French, Theorem 5.1 shows that our definition of I-diagrams is consistent with Postnikov's definition.

Now let $(W, j)=\left(B_{n}, n\right)$ so that $O_{w} \subset Q^{j}$ can be identified with a shape (a lower order ideal) within a staircase of size $n$. We refer to the $n$ boxes along the diagonal of $Q^{j}$ as the diagonal boxes.

Theorem 5.3 A type $\left(B_{n}, n\right)$ I-diagram is an $\oplus$-diagram $D$ of shape $O_{w}$ such that

1. if there is $a 0$ above (and in the same column as) $a+$ then all boxes to the left and in the same row as that 0 must also be 0 's.

2. any diagonal box containing a 0 must have only 0 's to the left of it.

Now let $(W, j)=\left(B_{n}, 1\right)$ so that $O_{w} \subset Q^{j}$ can be identified with a single row. We call the box labeled $n$ (if contained in $O_{w}$ ) the middle box, and any two boxes with the same simple label conjugate. The conjugate of the middle box is itself.

Theorem 5.4 A type $\left(B_{n}, 1\right) \mathrm{I}$-diagram is an $\oplus$-diagram $D$ of shape $O_{w}$ such that if there is a 0 to the right of the middle box, then the box $b$ immediately to the left of this 0 and the conjugate $b^{\prime}$ to $b$ cannot both contain + 's. 
Now let $(W, j)=\left(D_{n}, n\right)$ so that $O_{w} \subset Q^{j}$ can be identified with a shape contained inside a staircase. We refer to the $n$ boxes along the diagonal of $Q^{j}$ as the diagonal boxes. The distance of a box $b$ from the diagonal is the number of boxes that $b$ is on top of, so that a diagonal box has distance 0 from the diagonal.

In the following we will say that a box $b$ is to the left or right (above or below) another $b^{\prime}$ if and only if they are also in the same row (column). We will use compass directions when the same row or column condition is not intended.

Theorem 5.5 A type $\left(D_{n}, n\right)$ I-diagram is an $\oplus$-diagram $D$ of shape $O_{w}$ such that

1. if there is a 0 above $a+$ then all boxes to the left of that 0 must also be 0 's.

2. if there is a 0 with distance $d$ from the diagonal to the right of $a+$ in box $b$ then there is no + strictly southwest of $b$ and $d+1$ rows south of the 0 .

3. one cannot find a box containing a 0 and three distinct boxes $b_{1}, b_{2}, b_{3}$ containing + 's so that $c$ has distance $d$ from the diagonal and is to the right of $b_{1}$, the box $b_{2}$ is the box $d+1$ rows below $b_{1}$, and finally $b_{3}$ is strictly northwest of $b_{2}$ and strictly south of $b_{1}$.

Now let $(W, j)=\left(D_{n}, 1\right)$ so that $O_{w} \subset Q^{j}$ can be identified with a shape contained inside the doubled tail diamond of size $n$. We call the boxes labeled $n-1$ and $n$ (if contained in $O_{w}$ ) the middle boxes, and any two boxes with the same simple label conjugate. If $b$ is a middle box then the conjugate of $b$ is the other middle box.

Theorem 5.6 A type $\left(D_{n}, 1\right)$ J-diagram is an $\oplus$-diagram $D$ of shape $O_{w}$ such that if there is a 0 in a box $c$ greater than the middle boxes, then for any box $b \lessdot c$ covered by $c$ the conjugate $b^{\prime}$ and the box $b$ itself cannot both contain +'s.

\section{Enumeration of cells}

The cells in the totally non-negative Grassmannian for type $A$ were enumerated in $(15 ; 8)$. In this section we will give some formulae and recurrences for the number of totally non-negative cells in Grassmannians of types $\left(B_{n}, 1\right),\left(D_{n}, 1\right),\left(B_{n}, n\right)$, and $\left(D_{n}, n\right)$. We will often count the cells which lie inside the open Schubert cell, or in other words, we will count I-diagrams of maximal shape.

Let $\hat{b}_{n}$ be the number of type $\left(B_{n}, 1\right)$ I-diagrams of maximal shape, and let $\hat{b}_{n}(q)$ be the $q$-generating function of $\left(B_{n}, 1\right) \mathrm{I}$-diagrams of maximal shape, where we weight $\mathrm{J}$-diagrams according to the number of +'s. Similarly define $\hat{d}_{n}$ and $\hat{d}_{n}(q)$. Below, $[i]:=1+q+q^{2}+\cdots+q^{i-1}$ denotes the $q$-analogue of $i$.

Proposition 6.1 $\hat{b}_{n}(q)$ is the coefficient of $x^{n}$ in

$$
\frac{1-\left(q+q^{2}\right) x+\left(1-q^{2}\right) x^{2}}{1-[2]^{2} x+[2] x^{2}} .
$$

In particular, the numbers $\hat{b}_{n}$ are equal to sequence A006012 in Sloane (13). 
Proposition 6.2 $\hat{d}_{n}(q)$ is the coefficient of $x^{n}$ in

$$
\frac{1-\left(q+q^{2}\right) x+\left(1-2 q^{2}-q^{3}\right) x^{2}+\left(1+2 q-q^{3}\right) x^{3}}{1-[2]^{2} x+[2] x^{2}} .
$$

In particular, the numbers $\hat{d}_{n}$ (for $n \geq 3$ ) are given by sequence A007070 in Sloane (13).

Let $B(n)$ be the number of type $\left(B_{n}, n\right)$ I-diagrams.

Proposition 6.3 The sequence of numbers $B(n)$ is sequence A010844 from Sloane (13). The numbers are given by the recurrence $B(0)=1$ and $B(n+1)=2(n+1) B(n)+1$.

Let $b(n)$ be the number of type $\left(B_{n}, n\right)$ J-diagrams of maximal (staircase) shape. Let $[i]^{(j)}$ denote the $j$-th derivative (with respect to $q$ ) of the $q$-analogue $[i]$.

Proposition 6.4 We have $b(0)=1$ and

$$
b(n)=[n+1] b(n-1)+q^{2} \sum_{i=1}^{n-2} \frac{[n-1]^{(i)}}{i !} b(n-i-1) .
$$

Let $\mathcal{B}_{n}$ denote the set of $\left(B_{n}, n\right)$ J-diagrams of maximal (staircase) shape such that the bottom square contains a + . The set of $\left(B_{n}, n\right) \mathrm{J}$-diagrams of maximal shape has twice the cardinality of $\mathcal{B}_{n}$, since the bottom square of a J-diagram imposes no restrictions on any other square.

Definition 6.5 A preference function of $n$ is a word of length $n$ such that there exists a $k \leq n$ such that all the numbers 1 through $k$ occur at least once.

Theorem 6.6 The set $\mathcal{B}_{n}$ is in bijection with the set of preference functions of length $n$. Therefore the number of maximal type $\left(B_{n}, n\right) \mathrm{J}$-diagrams has twice the cardinality of the preference functions of length $n$. This is sequence A000670 in the Sloane Encyclopedia of Integer Sequences (13).

A preference function is atomic if no strict leading subword consists of the only occurrences in the word of the letters 1 through $j<k$.

Theorem 6.7 Atomic preference functions of length $n$ are in bijection with maximal type $\left(D_{n}, n\right) \mathrm{I}-$ diagrams. These numbers are sequence A095989 in Sloane (13).

\section{References}

[1] A. Bjorner and F. Brenti, Combinatorics of Coxeter groups, Graduate Texts in Mathematics, 231, Springer, New York, 2005.

[2] G. Cauchon, Spectre premier de $0_{q}\left(M_{n}(k)\right)$ : image canonique et separation normale. (French) J. Algebra 260 (2003), no. 2, 519-569.

[3] S. Corteel and L. Williams, Tableaux combinatorics for the asymmetric exclusion process, Adv. Appl. Math., 39, (2007), 293-310. 
[4] V. Deodhar, On some geometric aspects of Bruhat orderings. I. A finer decomposition of Bruhat cells, Invent. Math. 79 (1985), no. 3, 499-511.

[5] T. Lam and M. Shimozono, Dual graded graphs for Kac-Moody algebras, Algebra and Number Theory, to appear; http://www.arxiv.org/abs/math.CO/0702090.

[6] G. Lusztig, Total positivity in partial flag manifolds, Representation Theory, 2 (1998), 70-78.

[7] R. Marsh and K. Rietsch, Parametrizations of flag varieties, Representation Theory, 8 (2004).

[8] A. Postnikov, Total positivity, Grassmannians, and networks, preprint, 2006; http://arxiv.org/abs/math/0609764.

[9] A. Postnikov, D. Speyer, and L. Williams, Matching polytopes, toric geometry, and the non-negative part of the Grassmannian, preprint, 2007; http://arxiv.org/abs/0706.2501.

[10] R. Proctor, Bruhat lattices, plane partition generating functions, and minuscule representations. European J. Combin. 5 (1984), no. 4, 331-350.

[11] K. Rietsch, Total positivity and real flag varieties, Ph.D. Dissertation, MIT, 1998.

[12] K. Rietsch and L. Williams, The non-negative part of $G / P$ is a CW complex, in preparation.

[13] N. Sloane, The On-Line Encyclopedia of Integer Sequences, published electronically at http://www.research.att.com/ njas/sequences/.

[14] J. Stembridge, On the Fully Commutative Elements of Coxeter Groups. J. Algebraic Combinatorics 5 (1996), 353-385.

[15] L. Williams, Enumeration of totally positive Grassmann cells, Adv. Math., 190 (2), 2005, 319-342. 


\begin{tabular}{|c|c|}
\hline Parabolic quotient & $Q^{j}$ \\
\hline$W=A_{n-1}$ & 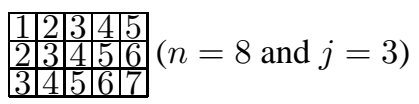 \\
\hline$W=B_{n}$ and $j=1$ & $112|3| 4|3| 2 \mid 10(n=4)$ \\
\hline$W=B_{n}$ and $j=n$ & 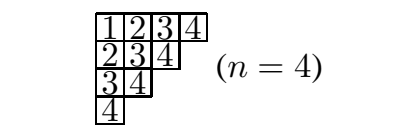 \\
\hline$W=D_{n}$ and $j=1$ & 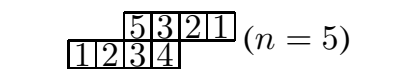 \\
\hline$W=D_{n}$ and $j=n$ & 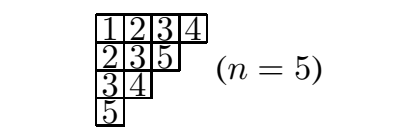 \\
\hline$W=E_{6}$ and $j=1$ & 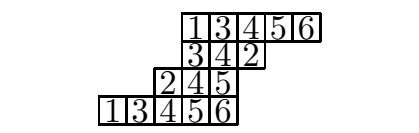 \\
\hline$W=E_{7}$ and $j=1$ & 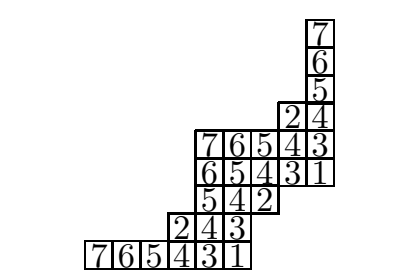 \\
\hline
\end{tabular}

Fig. 2: Underlying posets of parabolic quotients 
